\title{
SOCS2 affects the proliferation, migration, and invasion of nasopharyngeal carcinoma cells via regulating EphA1
}

\author{
B. Q. DAI ${ }^{1, *}, \mathrm{X} . \mathrm{ZHANG}^{2}$ \\ ${ }^{1}$ Department of Otorhinolaryngology, Cangzhou Central Hospital, Cangzhou, Hebei, China; ${ }^{2}$ First Department of Respiration, Cangzhou Cen- \\ tral Hospital, Cangzhou, Hebei, China \\ ${ }^{*}$ Correspondence: bao140@yeah.net
}

Received August 7, 2019 / Accepted September 30, 2019

\begin{abstract}
Although the incidence of nasopharyngeal carcinoma (NPC) is relatively low, the mortality is very high and the patients have a poor prognosis. Thus, it is urgent to find a novel biomarker and a new therapeutic strategy. Suppressor of cytokine signaling-2 (SOCS2) was reported to be associated with various malignancies. However, the exact role of SOCS2 in NPC still remains largely unsure. In the present study, we showed that the expression of SOCS2 was significantly upregulated in NPC patients and cells. And the high expression of SOCS2 predicted a worse outcome in NPC patients. Moreover, the in vivo experiments indicated that knockout of SOCS2 inhibits the proliferation, migration, and invasion of NPC cells. Besides, we found a positive relationship between SOCS2 and EphA1 in NPC tissues. The rescue experiments indicated that SOCS2 affected the malignancy of NPC cells by regulating the expression of EphA1. Altogether, our data uncovered the ontogenetic role of SOCS2 dysregulation during the tumorigenesis of NPC. SOCS2 might serve as a biomarker during the diagnosis and treatment of NPC. And targeting SOCS2 might provide a novel treatment strategy for NPC patients.
\end{abstract}

Key words: nasopharyngeal carcinoma, SOCS2, EphA1, carcinogenesis

Nasopharyngeal carcinoma (NPC) is an unfrequented tumor with a high ratio of death [1-3]. The incidence of NPC is relatively low and presents a distinct racial and geographic distribution $[4,5]$. Despite the progress achieved in many therapeutic strategies, the underlying mechanism of NPC still remains largely unknown and the prognosis of cancer patients is also relatively poor $[6,7]$. Thus, it is urgent to find a novel biomarker and explore the relevant mechanism.

The suppressor of cytokine signaling 2 (SOCS2) belongs to the SOCS family composed of SOCS1-7 and cytokine-inducible $\mathrm{SH} 2$-containing proteins $[8,9]$. They exert their function by suppressing the cytokine signal transduction and inhibiting cytokine-activated JAK/STAT $[10,11]$. The SOCS family plays pivotal roles in the regulation of immune responses and tumorigenesis [12]. Emerging studies demonstrated that the aberrant regulation of SOCS proteins was associated with the development of various cancers, including lung cancer [13]. A recent study reported that SOCS2 was significantly dysregulated in higher-grade samples of breast cancer [14]. Another study suggested that the expression of SOCS2 was upregulated by P53 dysfunction in colon cancer and associated with the potential tumorigenic ability [15]. However, the exact role of SOCS2 in lung cancer, especially in NP, still remains largely unsure.

The ephrin receptor of the protein-tyrosine kinase family plays a significant role in various physiological processes, including cell migration, angiogenesis, and vascular development [16]. Emerging studies demonstrated that abnormal expression of Eph receptor tyrosine kinases was associated with malignancies [17]. EphA1 belongs to the Eph family, and Ishikawa et al. suggested that higher expression of EphA1 was related to favorable clinicopathological features in lung cancer [18], indicating the pivotal role of EphA1 in tumorigenesis.

In the present study, we aimed to explore the potential functions of the SOCS2 during the NP malignancy and the underlying mechanism. By using the clinical sample of NP samples, it allowed us to verify the possibility of SOCS2 to be a biomarker during the diagnosis and treatment of NP.

\section{Patients and methods}

Ethics statement. The study was approved by the Ethics Committee for clinical research and complies with the 
tenets of the Declaration of Helsinki and the Institutional Review Board of the participating centers. Written informed consent for molecular genetic studies was obtained from all participants.

Patients. In this study, thirty NP specimens acquired from an equal number of patients who underwent surgical resection due to lung cancer were included. Institutional review board approval was obtained to use archived material for research purposes. None of the patients had received radiation or chemotherapy before surgery (Table 1).

Cell culture. The human NP cell lines NP69 (ATCC5859), CNE2 (ATCC-1434), CNE1 (ATCC-0364), HONE1 (ATCC-0369), HNE1 (ATCC-0366), and 6-10B (ATCC6605) were purchased from the Chinese Academy of Sciences Cell Bank. The cell lines except NP69 were cultured in RPMI 1640 medium (Flow Laboratories Irvine, Scotland) containing $10 \%$ sterile FBS, $1 \%$ penicillin and streptomycin. The cell line NP69 was cultured in keratinocyte/serum-free medium + EGF (epidermal growth factor). All the cell lines were incubated at $37^{\circ} \mathrm{C}$ in a humidified atmosphere under $5 \% \mathrm{CO}_{2}$ conditions.

Western blotting. For western blotting assay, cells were lysed using the RIPA buffer and protease inhibitor on ice for 25 minutes. Total proteins were collected by centrifugation at $14,000 \times \mathrm{g}$ at $4^{\circ} \mathrm{C}$ for $15 \mathrm{~min}$ and measured by BCA protein assay kit (Bio-Rad Laboratories, Hercules, CA, USA), then subjected to $12 \%$ SDS-PAGE and transferred to polyvinylidene difluoride (PVDF) membrane. The membrane was blocked with 5\% BSA for $1 \mathrm{~h}$ and incubated with primary SOCS2 (CST, \#2779), EphA1 (CST, \#90673), and GAPDH (CST, \#2118) antibodies overnight at $4{ }^{\circ} \mathrm{C}$. Specific primary antibodies were captured using secondary antibodies and were detected using the FluorChem M system (ProteinSimple, San Jose, CA, USA).

Quantitative real-time PCR (qRT-PCR). Total RNA was extracted with Trizol reagent (Takara, Japan) and RNA was purified with chloroform and isopropanol. SYBR Green Realtime PCR Master Mix (Toyobo, Osaka, Japan) and StepOnePlus Real-Time PCR Systems (Applied Biosystems) were used for polymerase chain reaction (PCR) referring to the manufacturer's instructions. GADPH was used as an internal standard. Relative expression was calculated according to $2^{-\triangle \Delta C T}$ method. Pairs of forward and reverse primer sets were used as follows: SOCS2 (forward: 5'-CAGATGTGCAAGGATAAGCGG-3' and reverse: 5'-GCGGTTTGGTCAGATAAAGGTG-3'), GAPDH (forward: 5'-GGAGCGAGATCCCTCCAAAAT-3' and reverse: 5'-GGCTGTTGTCATACTTCTCATGG-3'), SOCS2 sgRNA1 (HGLibB_46069-forward: 5'-CACCAAAGGTGAACAGTGCCGTTC-3' and HGLibB_46069-reverse: 5'-AAACGAACGGCACTGTTCACCTTT-3'), SOCS2 sgRNA2 (HGLibB_46070-forward: 5'-CACCTGCCTTGAGCCCTCCGGGAA-3' and HGLibB_46070-reverse: 5'-AAACTTCCCGGAGGGCTCAAGGCA-3'), SOCS2 Control (HGLibB_58021-forward: 5'-CACCGAGAAGT-
Table 1. Clinicopathological features of 30 patients with NPC.

\begin{tabular}{|c|c|c|c|}
\hline \multirow{2}{*}{ Characteristics } & \multicolumn{2}{|c|}{ SOCS2expression $^{\mathrm{a}}$} & \multirow{2}{*}{ p-value } \\
\hline & Low $^{\mathrm{b}}$ & High $^{c}$ & \\
\hline Sex & & & 0.713 \\
\hline Male & 7 & 6 & \\
\hline Female & 8 & 9 & \\
\hline Age, years & & & 0.464 \\
\hline$<55$ & 7 & 9 & \\
\hline$\geq 55$ & 8 & 6 & \\
\hline T-stage & & & 0.025 \\
\hline $\mathrm{T} 1-2$ & 9 & 3 & \\
\hline T3-4 & 6 & 12 & \\
\hline $\mathrm{N}$ stage & & & 0.001 \\
\hline No & 10 & 3 & \\
\hline $\mathrm{N} 1 / 2$ & 5 & 12 & \\
\hline Lymphatic metastasis & & & 0.001 \\
\hline Negative & 12 & 3 & \\
\hline Positive & 3 & 12 & \\
\hline
\end{tabular}

${ }^{\mathrm{a} C u t}$-off $=$ median SOCS2 expression. ${ }^{\mathrm{b}}$ Classified in 30 patients with expres-

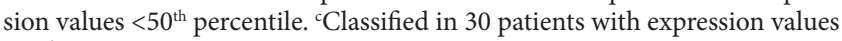
$>50^{\text {th }}$ percentile.

GGGGAGCCATTGG-3' and HGLibB_58021-reverse: 5'-AAACCCAATGGCTCCCCACTTCTC-3'), EphA1 (forward: 5'-AGATGGGTGGAGTGAACAGC-3' and reverse: 5'-CCTTGCATTGGGCAGTCCT-3').

CRISPR/Cas9 genome-editing system gene deletion. Lentiviral vectors expressing Cas9 and single guide RNAs (sgRNAs) targeting SOCS2 were constructed by Hanyin Co. (Shanghai, China). The guide sequences used were 5'-GACATCTACAGGTGCAATCG-3' for sgRNA1 and 5'AGAGGGAGTTCCATTGACC-3' for sgRNA2. Cells were then infected with the lentivirus. After infection for $72 \mathrm{~h}$, puromycin $(2 \mu \mathrm{g} / \mathrm{ml})$ was added for 3 days. After recovering for 4-6 days, individual colonies were picked. Contaminating gDNA was eliminated from isolated RNA using the Turbo DNA-free kit (Thermo Fisher Scientific). Western blot analysis was performed to confirm the lack of SOCS2 protein.

Overexpressed plasmid construction and siRNA. The SOCS2 and EphA1 overexpression vector versions were amplified by PCR from plasmid pGEX-4T-3-EpCAM. The primers used were primer-K (5'-GATTCGTTTTGGGGTTCTC-3') and primer-L (5'-TCAGCGTTCTTAGCGTT-3'). The siRNA of EphA1 was purchased from Thermo Scientific (\#146623, Massachusetts, USA).

Transwell migration and invasion assay. To evaluate the migration and invasion of CNE1 and 6-10B cells, Transwell chambers (24-well Transwell chambers, $8 \mu \mathrm{m}$ pore size; Corning, Steuben County, NY, USA) were pre-coated with fibronectin for migration assay or with Matrigel (BD Biosciences, Franklin Lakes, NJ, USA) for invasion assay. We seeded $5.0 \times 10^{4}$ cells in the upper chambers and the medium 
containing $10 \%$ FBS was placed into the lower chamber. After incubation for $24 \mathrm{~h}$ at $37^{\circ} \mathrm{C}$, cells on the upper surface were washed away, whereas the cells on the bottom surface were fixed with $4 \%$ paraformaldehyde for $10 \mathrm{~min}$ and stained with $0.2 \%$ crystal violet. The cells were imaged using a digital light microscope (Leica, Germany).

CCK-8 assay. Cell Counting Kit-8 (CCK-8, Yeasen, Shanghai, China) was used for examining cell viability according to the manufacturer's instruction. $6 \times 10^{3}$ cells/ well were seeded in a 96 -well plate and incubated at $37^{\circ} \mathrm{C}$ with $5 \% \mathrm{CO}_{2}$ for $48 \mathrm{~h}$. During detection, $100 \mu \mathrm{l}$ of fresh medium containing $10 \mu \mathrm{l}$ of CCK-8 was added to each well and incubated for $1 \mathrm{~h}$. After incubation, the absorbance was measured at $450 \mathrm{~nm}$ by a microplate reader from Thermo Scientific (Massachusetts, USA).

Statistical analysis. Three independent experiments were performed in triplicate. All data are reported as the mean \pm SD or median (interquartile range). A p-value $<0.05$ was considered the limit of statistical significance. SPSS 19.0 for Windows Software was used for all analyses.

\section{Results}

SOCS2 is an important gene in the carcinogenesis of NP. Previously research has revealed that SOCS2 plays a key role in the carcinogenesis of various kinds of tumors $[10,11]$. In order to determine the function of SOCS2 in NP, we assessed the expression level of SOCS2 in the 30 pair-matched normal tissues and NP tissues by qRT-PCR. Compared to the normal tissues, SOCS2 was highly expressed in NP tissues at the transcript level (Figure 1A). In addition, we analyzed the Kaplan-Meier survival curve of $30 \mathrm{NP}$ patients, the results indicated that higher expression of SOCS2 resulted in short overall survival time (Figure 1B). Next, we determined the level of SOCS2 in the human nasopharyngeal epithelial cells (NP-69) and NP cell lines. As illustrated in Figure 1C and Figure 1D, SOCS2 was strongly elevated in NP cells at both mRNA and protein levels. These results imply that SOCS2 may be a critical regulator in the development of NP.

Knock out of SOCS2 inhibits the proliferation, migration, and invasion of NP cells. As SOCS2 was upregulated in NP, to determine the detailed role of SOCS2, we depleted the level of SOCS2 in two NP cell lines (CNE1 and 6-10B) by using the Crispr/cas9 system. The depletion efficiency of SOCS2 was confirmed by western blot (Figure 2A). Functionally, proliferation assay demonstrated that knock out of SOCS2 resulted in a dramatic reduction in the growth rate of CNE1 and 6-10B cells. (Figures 2B, 2C). Moreover, transwell migration and invasion assay were applied to assess the metastatic ability of NP cells. The number of cells passed through the transwell filters coated without (migration) or with (invasion) matrigel was lower in SOCS2 depleted cells when compared to the control cells (Figure 2D-2I).

The upregulation of SOCS2 promotes the proliferation, migration, and invasion of NP cells. To further investigate the functional significance of SOCS2 in NP, we constructed the overexpression plasmid of SOCS2 and transfected CNE1 and 6-10B cells, which led to a significant upregulation of SOCS2 expression (Figure 3A). Overexpression of SOCS2 in $\mathrm{CNE} 1$ and 6-10B cell lines resulted in a markedly increased cell growth rate, as measured by CCK- 8 assay (Figures 3B, 3C). To reveal the impact of SOCS2 on the metastasis, transwell assays were performed in NP cell lines. In line with the results of proliferation, cells overexpressed with SOCS2 presented a higher transfer capability, a significant in cell migration and invasion was observed in SOCS2 up-regulated group (Figures 3D-3I).

SOCS2 regulates the expression of EphA1 in NP. It has been reported that the upregulation of SOCS2 could regulate the level of EphA2 by direct and indirect mechanisms $[10,11]$, so we hypothesize that EphA1 in NP cells may also be regulated by SOCS2. We detected the expression of EphA1 in SOCS2 knock out group, SOCS2 overexpression group and negative control group, the expression level of EphA1 was suppressed in SOCS2 knock out group, on the contrary, the expression of EphA1 was remarkably upregulated in the SOCS2 overexpression group at both protein and mRNA levels (Figures 4A, 4B). In addition, the relationship between SOCS2 and EphA1 was determined in NP tissues via qRT-PCR with previously used 30 pair-matched samples. The data suggested that the level of SOCS2 was positively correlated with the expression of EphA1 in NP tissues (Figure 4C).

SOCS2 affects the malignancy of NP cells through the regulation of EphA1. To determine whether EphA1 mediated the oncogenic role of SOCS2 in NP cells, overexpression plasmid and siRNA of EphA1 were chemically synthesized and transfected into NP cell lines. The transfection efficiency was confirmed by western blot (Figures 4D, $4 \mathrm{G})$. The results demonstrated that increasing the expression of EphAl could promote the migration and invasion of NP cells. Notably, knock out of SOCS2 mediated suppression of the metastasis of NP cells could be reversed by co-expression of EphA1 overexpression plasmid (Figures 4E, 4F; Suppl. Figures S1A, S1B). In addition, the downregulation of EphA1 impairs the migration and invasion of NP cells. And the oncogenic effect of SOCS2 on migration and invasion was neutralized by knockdown of EphA1 (Figures 4H, 4I; Suppl. Figures S1C, S1D). Notably, little effect of EphA1 was found on the survival of NP cells (data not shown). Therefore, the above data indicated that SOCS2 regulate the metastasis of NP cells by affecting the expression of EphA1.

\section{Discussion}

SOCS family proteins are initially identified to be associated with multiple signaling pathways, such as JAK/STAT signaling, insulin signaling, and NF-kappaB pathway [19-21]. Among the SOCS family, the studies focused on SOCS2 are relatively limited. The regulation of SOCS2 

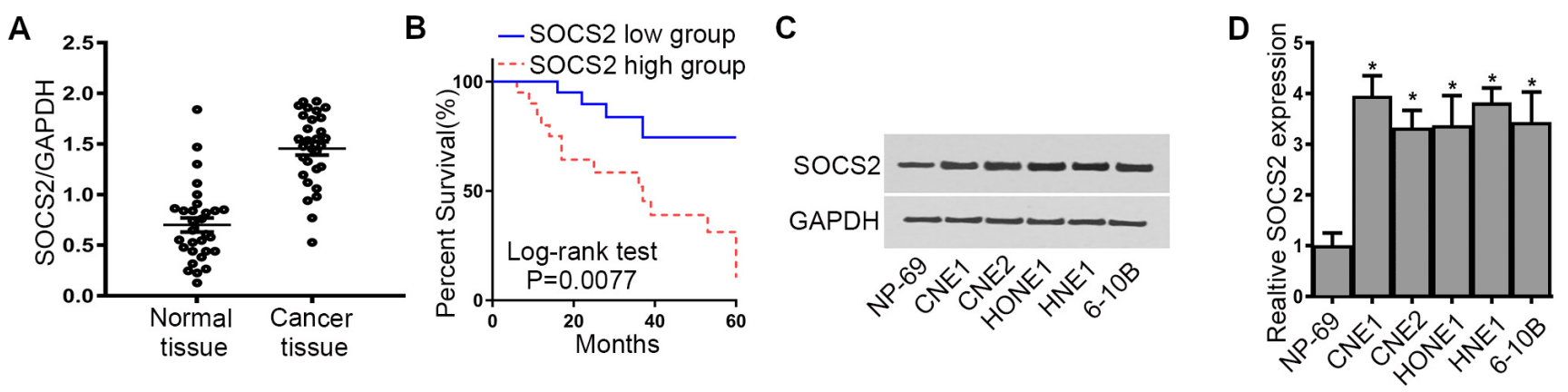

Figure 1. SOCS2 was increased in NP tissues and cell lines. A) The expression level of Rab5 in NP tissues and pair-matched normal tissues was detected by qRT-PCR. B) Analysis of overall survival of NP patients with different SOCS2 expression. C, D) Relative expression of SOCS2 in NP cells was measured by $\mathrm{qRT}-\mathrm{PCR}$ and western blot. ${ }^{*} \mathrm{p} \leq \mathbf{0 . 0 5}$
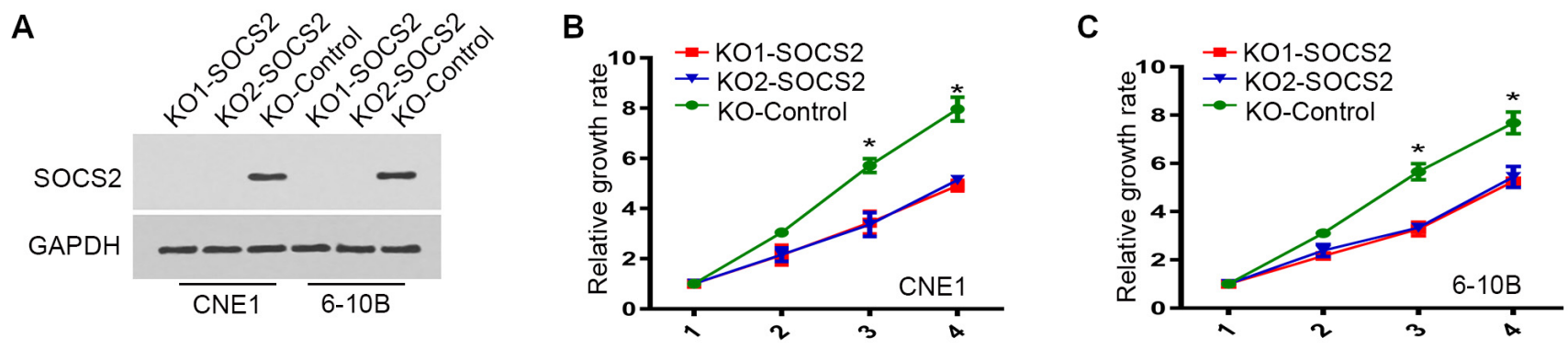

D

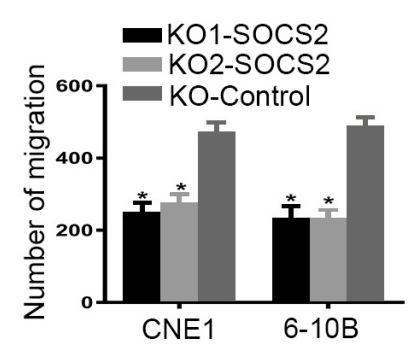

G

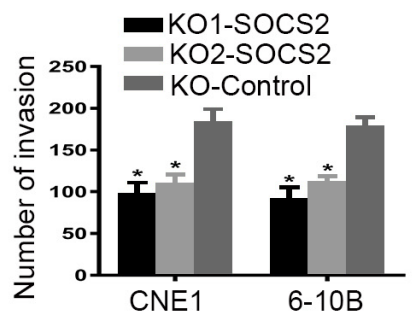

E

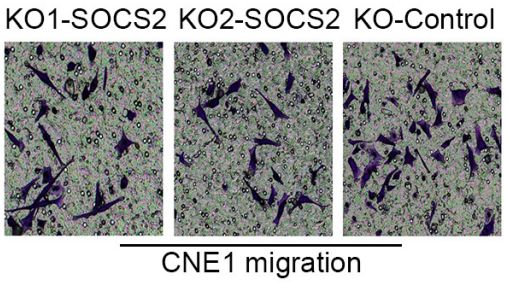

H

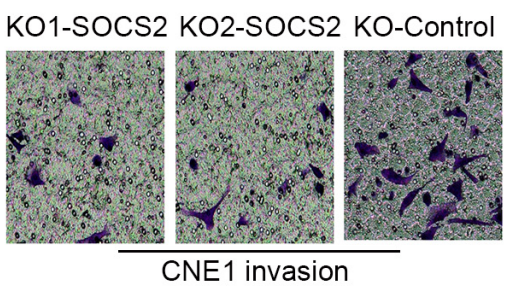

F

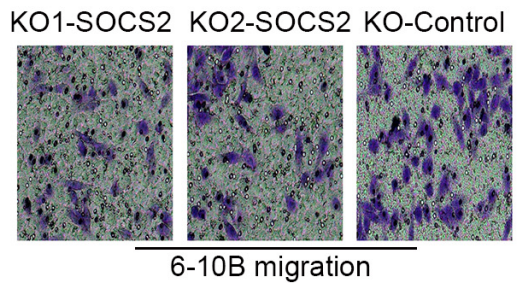

I

KO1-SOCS2 KO2-SOCS2 KO-Control

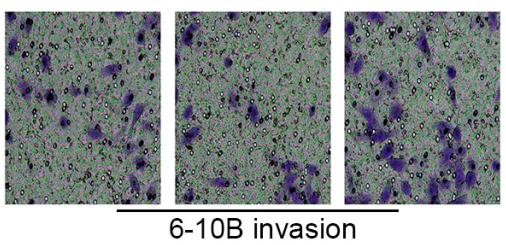

Figure 2. Knockdown of SOCS2 inhibited the proliferation, migration, and invasion of NP cells. A) The knock out of SOCS2 by the Crispr/Cas9 system. B, C) The proliferation rate of NP cells was measured by CCK-8 assay. D-I) Migration and invasion ability of NP cells were assessed by transwell assays. ${ }^{*} \mathrm{p} \leq 0.05$

involved in several factors, including growth hormone, interleukins, and erythropoietin [22-24].

As for the functions of SOCS2 in cancers, Cui et al. previously reported that overexpression of SOCS2 could inhibit tumor metastasis in hepatocellular carcinoma [25], and Zhao et al. reported that the downregulation of SOCS2 predicted poor prognosis of patients in human laryngeal squamous cell carcinoma [26]. Generally, the downregulation of SOCS2 is suggested to be related to a poor clinical outcome in various cancers. 
A

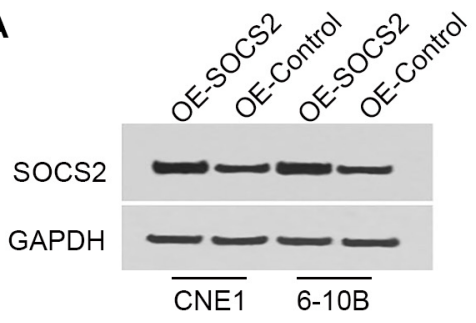

D

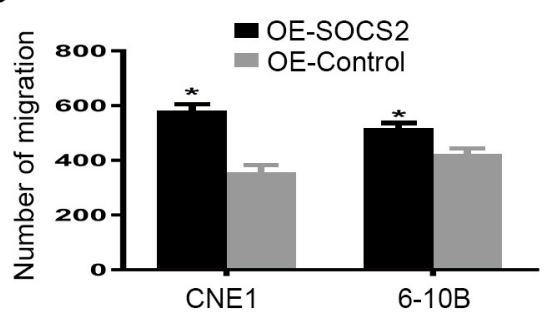

G

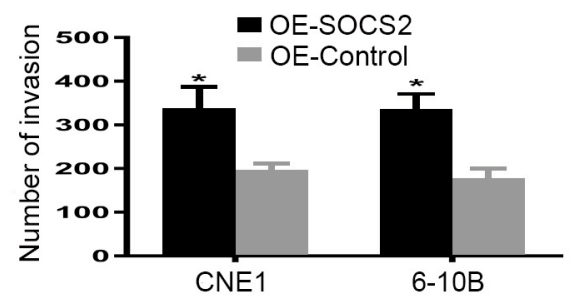

B

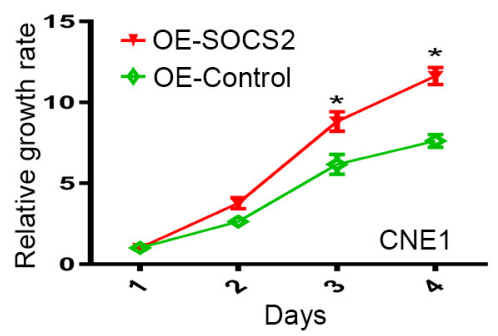

E

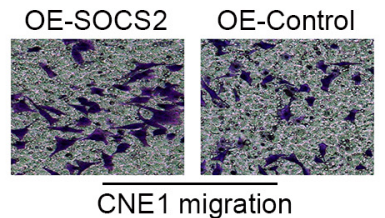

H

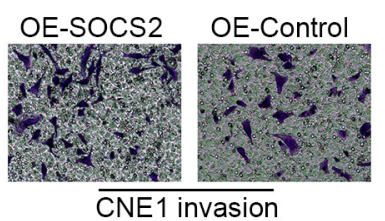

C

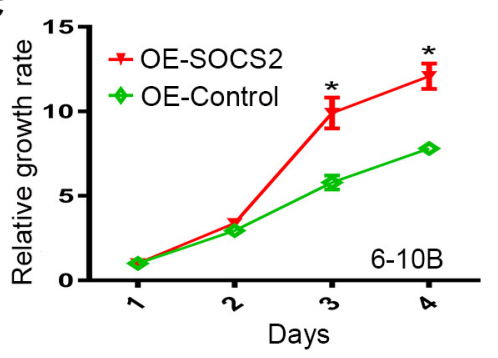

F

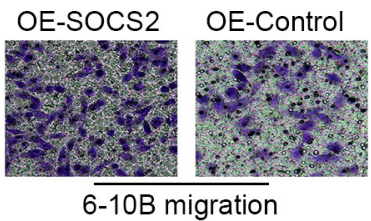

I

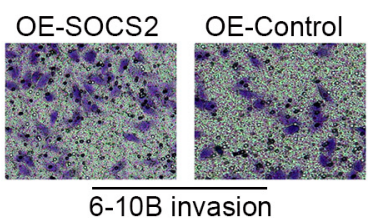

Figure 3. Overexpression of SOCS2 promoted the proliferation, migration, and invasion of NP cells. A) The expression of SOCS2 was effectively increased by SOCS2 overexpression plasmid. B, C) The proliferation rate of NP cells was measured by CCK-8 assay. D-I) Migration and invasion ability of NP cells were assessed by transwell assays. ${ }^{*} \mathrm{p} \leq 0.05$

However, recent studies reported a conflicting role of SOCS2 during carcinogenesis. Hoefer et al. reported that SOCS2 expression was increased in prostate cancer and also correlated with AR expression in the malignant tissue of patients [27]. Besides, SOCS2 was also identified as an oncogenic factor in chronic myeloid leukemia and anal cancer $[28,29]$. Here, we firstly present a concept of SOCS2 as a growth promoter during the carcinogenesis of NPC.

In the present study, we detected the expression of SOCS2 using IHC in 30 pairs of NPC patients. The results indicated that SOCS2 was highly expressed in NPC and the upregulated expression of SOCS2 predicted a worse outcome in NPC patients. Furthermore, we also found that SOCS2 knockdown led to a significant decrease in cell proliferation and invasion in NPC cells. By contrast, overexpression of SOCS2 facilitated the proliferation and invasion of NPC cells. Finally, we also proved a close correlation between SOCS2 and EphA1. By rescue experiments, we found that SOCS2 promoted the malignancy of NPC through regulating EphA1.

The controversial roles of SOCS2 during the tumorigenesis (promoter or inhibitor) were demonstrated by various studies. However, the deep reason to explain this phenomenon still remains unsure. Hoefer et al. presented a theory that the dual roles of SOCS2 depended on its concentration [27]. The relatively lower expression of SOCS2 inhibits GH, prolactin, and IL signaling while the higher expression of SOCS2 enhances the responsiveness to the indicated factors $[30,31]$. In our research, we found that the expression of SOCS2 in NPC was at high levels and SOCS2 functioned as a positive regulator during tumor proliferation.

The Ephrin family consisted of two subfamilies, including EphA and EphB. Emerging studies suggested that EphA1 was associated with malignancies and prognosis. EphA1 has already been identified as oncoproteins in various cancers. Here, we suggested that SOCS2 promotes the malignancy of NPC through regulating EphA1.

In conclusion, our study proved that SOCS2 promoted tumorigenesis of NPC by regulating EphA1 and overexpressed SOCS2 facilitated NPC cells proliferation in vivo and in vitro. SOCS2 might serve as a novel biomarker during the diagnosis and prognosis, and represents a new therapeutic strategy for the treatment of NPC. 
A

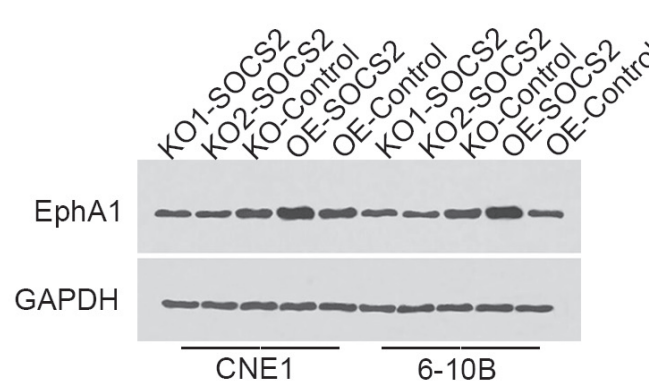

B

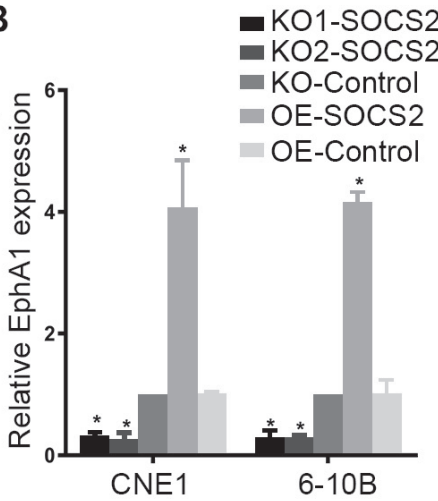

E

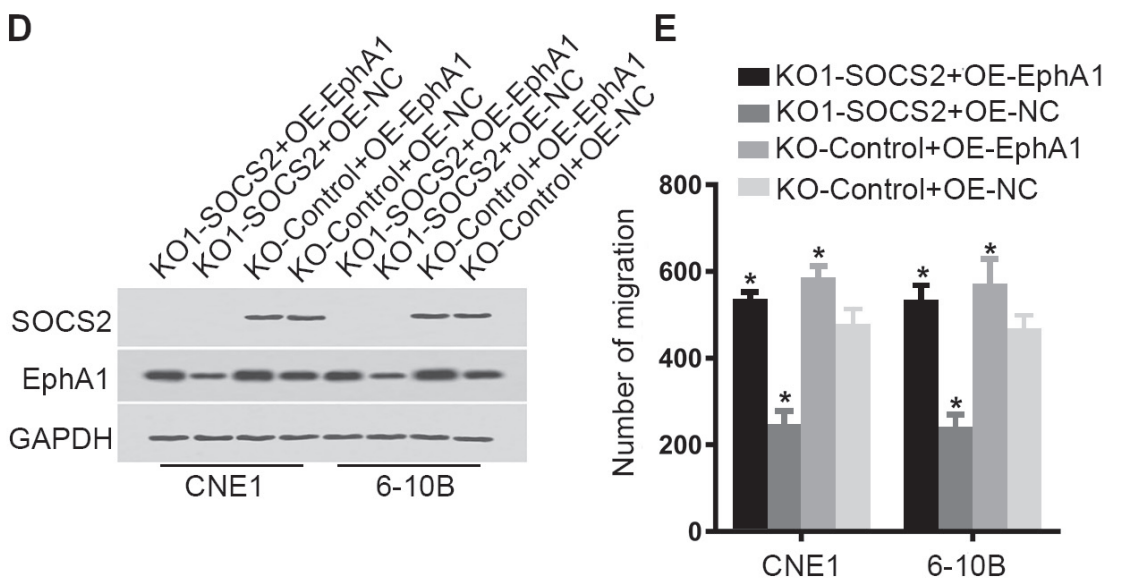

G

H

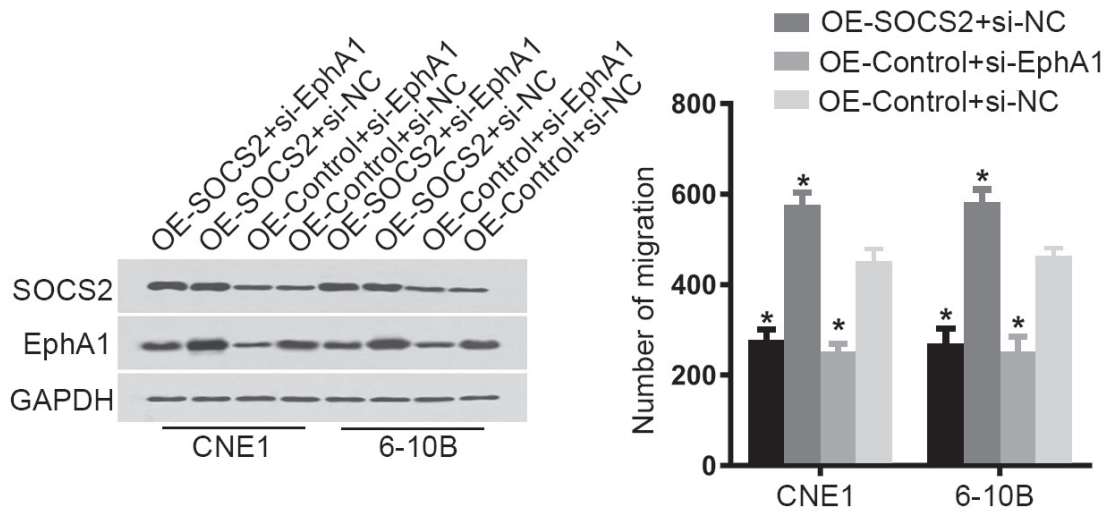

C

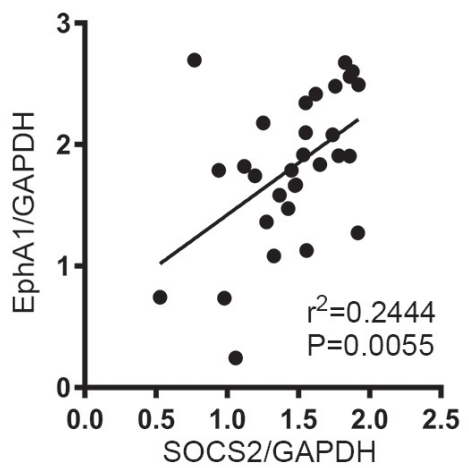

$\mathbf{F}$

-KO1-SOCS2+OE-EphA1 KO1-SOCS2+OE-NC KO-Control+OE-EphA1
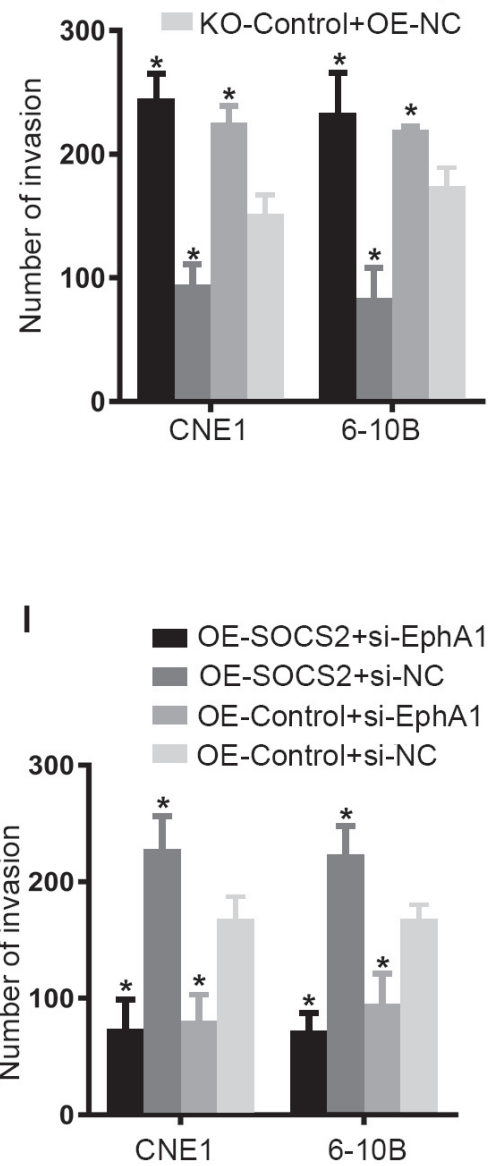

Figure 4. SOCS2 affects the malignancy of NP cells through the regulation of EphA1. A, B) The expression of EphA1 was measured by western blot and qRT-PCR. C) Correlation between SOCS2 and EphA1 was assessed by qRT-PCR. ${ }^{\star} p \leq 0.05$ D, G) The expression level of SOCS2 and EphA1 was measured by western blot. E, H) The migration ability of NP cells was assessed by transwell assays. F, I) The invasion ability of NP cells was assessed by transwell assays. ${ }^{*} \mathrm{p} \leq 0.05$ 
Supplementary information is available in the online version of the paper.

\section{References}

[1] PASTOR M, LOPEZ POUSA A, DEL BARCO E, PEREZ SEGURA P, ASTORGA BG et al. SEOM clinical guideline in nasopharynx cancer (2017). Clin Transl Oncol 2018; 20: 84-88. https://doi.org/10.1007/s12094-017-1777-0

[2] JIANG C, CHEN J, XIE S, ZHANG L, XIANG Y et al. Evaluation of circulating EBV microRNA BART2-5p in facilitating early detection and screening of nasopharyngeal carcinoma. Int J Cancer 2018; 143: 3209-3217. https://doi.org/10.1002/ ijc.31642.

[3] LAM WKJ, CHAN JYK. Recent advances in the management of nasopharyngeal carcinoma. F1000Res 2018; 7: F10001829. https://doi.org/10.12688/f1000research.15066.1

[4] SUN L, SONG J, HUANG Q. Clinicopathological and prognostic significance of 16 protein in nasopharynx cancer patients: A PRISMA-compliant meta-analysis. Medicine (Baltimore) 2019; 98: e14602. https://doi.org/10.1097/ MD.0000000000014602

[5] NIKITOVIC M, POPOVIC-VUKOVIC M, STANIC D, BOKUN J, PARIPOVIC L et al. Treatment outcome of childhood nasopharyngeal carcinoma: A single institution experience. Int J Pediatr Otorhinolaryngol 2018; 113: 168-172. https://doi.org/10.1016/j.ijporl.2018.07.031

[6] MAHDAVIFAR N, GHONCHEH M, MOHAMMADIAN-HAFSHEJANI A, KHOSRAVI B, SALEHINIYA $\mathrm{H}$. Epidemiology and Inequality in the Incidence and Mortality of Nasopharynx Cancer in Asia. Osong Public Health Res Perspect 2016; 7: 360-372. https://doi.org/10.1016/j. phrp.2016.11.002

[7] HE H, LIAO X, YANG Q, LIU Y, PENG Y et al. MicroRNA-494-3p Promotes Cell Growth, Migration, and Invasion of Nasopharyngeal Carcinoma by Targeting Sox7. Technol Cancer Res Treat 2018; 17: 1533033818809993. https://doi. org/10.1177/1533033818809993

[8] LETELLIER E, HAAN S. SOCS2: physiological and pathological functions. Front Biosci (Elite Ed) 2016; 8: 189-204.

[9] TURNLEY AM. Growth hormone and SOCS2 regulation of neuronal differentiation: possible role in mental function. Pediatr Endocrinol Rev 2005; 2: 366-371.

[10] BULATOV E, MARTIN EM, CHATTERJEE S, KNEBEL A, SHIMAMURA $S$ et al. Biophysical studies on interactions and assembly of full-size E3 ubiquitin ligase: suppressor of cytokine signaling 2 (SOCS2)-elongin BC-cullin 5-ring box protein 2 (RBX2). J Biol Chem 2015; 290: 4178-4191. https:// doi.org/10.1074/jbc.M114.616664

[11] ZHANG MY, FUNG TK, CHEN FY, CHIM CS. Methylation profiling of SOCS1, SOCS2, SOCS3, CISH and SHP1 in Philadelphia-negative myeloproliferative neoplasm. J Cell Mol Med 2013; 17: 1282-1290. https://doi.org/10.1111/ jcmm.12103
[12] SLATTERY ML, LUNDGREEN A, HINES LM, TORRESMEJIA G, WOLFF RK et al. Genetic variation in the JAK/ STAT/SOCS signaling pathway influences breast cancer-specific mortality through interaction with cigarette smoking and use of aspirin/NSAIDs: the Breast Cancer Health Disparities Study. Breast Cancer Res Treat 2014; 147: 145-158. https://doi.org/10.1007/s10549-014-3071-y

[13] ZHOU Y, ZHANG Z, WANG N, CHEN J, ZHANG X et al. Suppressor of cytokine signalling-2 limits IGF1R-mediated regulation of epithelial-mesenchymal transition in lung adenocarcinoma. Cell Death Dis 2018; 9: 429. https://doi. org/10.1038/s41419-018-0457-5

[14] GHAFOURI-FARD S, OSKOOEI VK, AZARI I, TAHERI M. Suppressor of cytokine signaling (SOCS) genes are downregulated in breast cancer. World J Surg Oncol 2018; 16: 226. https://doi.org/10.1186/s12957-018-1529-9

[15] KIM JH, LEE MJ, YU GR, KIM SW, JANG KY et al. Alterations in the p53-SOCS2 axis contribute to tumor growth in colon cancer. Exp Mol Med 2018; 50: 3. https://doi. org/10.1038/s12276-017-0001-1

[16] GROSSMAN EN, GIURUMESCU CA, CHISHOLM AD. Mechanisms of ephrin receptor protein kinase-independent signaling in amphid axon guidance in Caenorhabditis elegans. Genetics 2013; 195: 899-913. https://doi.org/10.1534/ genetics.113.154393

[17] LIERSCH-LOHN B, SLAVOVA N, BUHR HJ, BENNANIBAITI IM. Differential protein expression and oncogenic gene network link tyrosine kinase ephrin B4 receptor to aggressive gastric and gastroesophageal junction cancers. Int J Cancer 2016; 138: 1220-1231. https://doi.org/10.1002/ ijc. 29865

[18] ISHIKAWA M, MIYAHARA R, SONOBE M, HORIUCHI M, MENNJU T et al. Higher expression of EphA2 and ephrin-A1 is related to favorable clinicopathological features in pathological stage I non-small cell lung carcinoma. Lung Cancer 2012; 76: 431-438. https://doi.org/10.1016/j.lungcan.2011.12.004

[19] RYO A, SUIZU F, YOSHIDA Y, PERREM K, LIOU YC et al. Regulation of NF-kappaB signaling by Pin1-dependent prolyl isomerization and ubiquitin-mediated proteolysis of p65/ RelA. Mol Cell 2003; 12: 1413-1426. https://doi.org/10.1016/ s1097-2765(03)00490-8

[20] RUI L, YUAN M, FRANTZ D, SHOELSON S, WHITE MF. SOCS-1 and SOCS-3 block insulin signaling by ubiquitinmediated degradation of IRS1 and IRS2. J Biol Chem 2002; 277(44): 42394-8. https://doi.org/10.1074/jbc.C200444200

[21] LIU CZ, HE AY, CHEN LQ, LIMBU SM, WANG YW et al. Molecular characterization and immune response to lipopolysaccharide (LPS) of the suppressor of cytokine signaling (SOCS)-1, 2 and 3 genes in Nile tilapia (Oreochromis niloticus). Fish Shellfish Immunol 2016; 50: 160-167. https://doi. org/10.1016/j.fsi.2016.01.027

[22] GREENHALGH CJ, METCALF D, THAUS AL, CORBIN JE, UREN R et al. Biological evidence that SOCS-2 can act either as an enhancer or suppressor of growth hormone signaling. J Biol Chem 2002; 277: 40181-40184. https://doi. org/10.1074/jbc.C200450200 
[23] RICO-BAUTISTA E, FLORES-MORALES A, FERNANDEZ-PEREZ L. Suppressor of cytokine signaling (SOCS) 2, a protein with multiple functions. Cytokine Growth Factor Rev 2006; 17: 431-439. https://doi.org/10.1016/j.cytogfr.2006.09.008

[24] WANG L, ZHANG Z, ZHANG R, HAFNER MS, WONG $\mathrm{HK}$ et al. Erythropoietin up-regulates SOCS2 in neuronal progenitor cells derived from SVZ of adult rat. Neuroreport 2004; 15: 1225-1229. https://doi.org/10.1097/01. wnr.0000127636.15181.c1

[25] CUI M, SUN J, HOU J, FANG T, WANG X et al. The suppressor of cytokine signaling 2 (SOCS2) inhibits tumor metastasis in hepatocellular carcinoma. Tumour Biol 2016; 37: 13521-13531. https://doi.org/10.1007/s13277-016-5215-7

[26] ZHAO X, ZHANG W, JI W. miR-196b is a prognostic factor of human laryngeal squamous cell carcinoma and promotes tumor progression by targeting SOCS2. Biochem Biophys Res Commun 2018; 501: 584-592. https://doi.org/10.1016/j. bbrc.2018.05.052

[27] HOEFER J, KERN J, OFER P, EDER IE, SCHÄFER G et al. SOCS2 correlates with malignancy and exerts growth-promoting effects in prostate cancer. Endocr Relat Cancer 2014; 21: 175-187. https://doi.org/10.1530/ERC-13-0446
[28] ARANY I, MULDROW M, TYRING SK. The endogenous interferon system in anal squamous epithelial lesions with different grades from HIV-positive individuals. Int J STD AIDS 2001; 12: 229-233. https://doi. org/10.1258/0956462011922977

[29] SUTHERLAND KD, LINDEMAN GJ, CHOONG DY, WITTLIN S, BRENTZELL L et al. Differential hypermethylation of SOCS genes in ovarian and breast carcinomas. Oncogene 2004; 23: 7726-7733. https://doi.org/10.1038/sj.onc.1207787

[30] TANNAHILL GM, ELLIOTT J, BARRY AC, HIBBERT L, CACALANO NA et al. SOCS2 can enhance interleukin-2 (IL-2) and IL-3 signaling by accelerating SOCS3 degradation. Mol Cell Biol 2005; 25: 9115-9126. https://doi.org/10.1128/ MCB.25.20.9115-9126.2005

[31] PIESSEVAUX J, LAVENS D, MONTOYE T, WAUMAN J, CATTEEUW D et al. Functional cross-modulation between SOCS proteins can stimulate cytokine signaling. J Biol Chem 2006; 281: 32953-32966. https://doi.org/10.1074/jbc. M600776200 